\section{Les vésicules extracellulaires}

Actrices de la communication entre les cellules du système nerveux

\author{
Élodie Leroux, Romain Perbet, Luc Buée, \\ Morvane Colin
}

blissent. Cette revue fournit un aperçu des différents rôles joués par les $V \varepsilon$ dans un cerveau sain lors du renforcement des réseaux par exemple, mais également dans un cerveau malade où les VE participent, entre autres, à la progression des maladies neurodégénératives et tumorales. <

\section{Vésicules extracellulaires et homéostasie cérébrale}

Dans le cerveau, un organe très dense où la mobilité cellulaire est restreinte, neurones et cellules gliales (microglie, oligodendrocytes, astrocytes) sécrètent des vésicules extracellulaires (VE) pour communiquer. Examinons, tout d'abord, comment les différentes populations cellulaires peuplant le cerveau utilisent ces VE pour garantir l'homéostasie cérébrale lors du développement, de la transmission synaptique ou encore de la myélinisation axonale (Figure 1, panneau supérieur).

\section{Développement du système nerveux central et des réseaux}

Le développement du système nerveux central (SNC) implique des processus de multiplication, de migration et de différenciation cellulaires parfaitement organisés. Cela se fait par un jeu d'expression de multiples facteurs de transcription échangés entre les cellules du SNC en développement. Le rôle des VE dans le transport de ces différentes molécules semble important. Par

Vignette (๔ Guillaume van Niel, Aurélie di Cicco, Graça Raposo, Daniel Levy). exemple, des $V \varepsilon$ issues de cultures neuronales dérivées de cellules souches pluripotentes humaines (hIPSc) induisent la prolifération et la différenciation de ces cellules en culture. Une analyse protéomique de ces $V \varepsilon$ a révélé la présence de facteurs impliqués dans ces deux processus, de même que dans la migration cellulaire [1]. Les astrocytes, tout comme les cellules microgliales, fournissent également un support trophique aux neurones. Les $V \varepsilon$ astrocytaires peuvent exposer à leur surface la protéine synapsine-l qui se lie au récepteur neuronal NCAM (neuronal cell adhesion molecule). L'internalisation de cette protéine après sa fixation à son récepteur par les neurones va promouvoir la survie neuronale et favoriser la croissance neuritique [2]. En réponse à l'ATP, des protéines impliquées dans la croissance neuritique, la ramification dendritique, la régulation de la transmission synaptique et la promotion de la survie neuronale, sont également sécrétées dans des $V \varepsilon$ astrocytaires [3]. Enfin, les VE microgliales, de par leur contenu en protéines associées à la survie et à la croissance, peuvent aussi activer la croissance neuritique [4].

\section{Modulation de l'activité électrique neuronale}

Les neurones sont les cellules les plus connues et étudiées du SN. Ils établissent entre eux plusieurs dizaines de milliers de connexions, appelées synapses, qui transmettent des informations via des signaux électriques. Ces signaux sont à l'origine d'une libération par le neurone pré-synaptique de neurotransmetteurs, excitateurs ou inhibiteurs, qui se lient à des récepteurs exprimés par le neurone post-synaptique. Cette liaison permet une entrée de calcium dans le neurone post- 

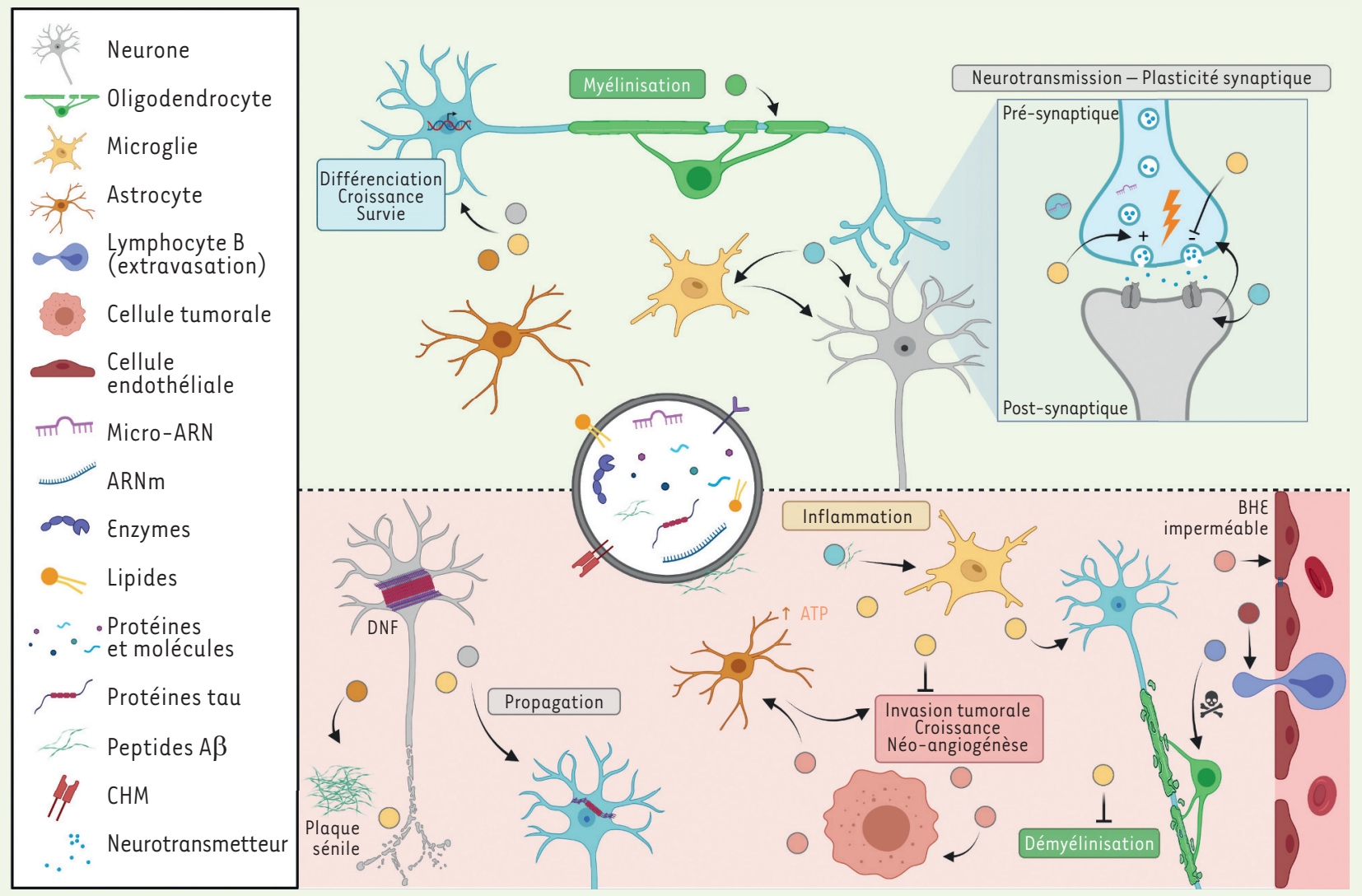

Figure 1. Les différents rôles des vésicules extracellulaires dans le système nerveux central. Le panneau supérieur (fond vert) représente les conditions physiologiques, le panneau inférieur (fond rouge) représente les conditions pathologiques. La diversité du contenu vésiculaire est illustrée dans la vésicule centrale. La couleur des VE dépend de leur origine cellulaire. BHE : barrière hémato-encéphalique ; CMH : complexe majeur d’histocompatibilité ; DNF : dégénérescence neurofibrillaire (figure réalisée avec BioRender.com).

synaptique et l'activation de seconds messagers capables de l'activer ou de l'inhiber. En réponse à une activité électrique excitatrice, les neurones sécrètent des $V \mathcal{E}$ qui seront internalisées par d'autres neurones [5-7]. Les $V \varepsilon$ microgliales semblent pouvoir réguler l'équilibre entre excitation et inhibition de la neurotransmission, soit en stimulant l'activité électrique neuronale excitatrice [8], soit en bloquant la transmission de l'information via l'activation de récepteurs présynaptiques exprimés par des neurones inhibiteurs [9].

Le couplage entre activité synaptique et libération vésiculaire pourrait également être utilisé dans des processus associés à la plasticité synaptique [10]. La capacité des synapses à être modifiées après stimulation permet la formation et le renforcement de circuits neuronaux garantissant notamment nos capacités d'associations et de mémoire. Cette plasticité synaptique dépend de signaux antérogrades (neurones pré- vers post-synaptiques) et rétrogrades (neurones post- vers présynaptiques) qui pourraient être véhiculés par les VE. Par exemple, la synaptotagmine 4 , une protéine impliquée dans la plasticité synaptique, est transportée de façon antérograde par les VE. Ce transport induit alors des signaux rétrogrades nécessaires à l'ajustement de l'activité du neurone pré-synaptique et à la croissance synaptique [11]. Les synapses actives peuvent également libérer des $V \varepsilon$ qui iront stimuler la microglie pour phagocyter les synapses inactives et donc promouvoir leur élimination [12]. La plasticité synaptique implique également des modifications des synapses, transitoires ou durables, suite à des changements de l'expression de certains gènes [13]. Ces changements nécessitent des trafics d'ARN messagers (ARNm) qui peuvent être contenus dans les VE [14]. Des micro-ARN, également contenus dans les $V \varepsilon$, peuvent participer à ces modifications, en régulant l'expression des ARNm dans la cellule réceptrice. En réponse à une activité électrique, des micro-ARN contenus dans les neurones pré-synaptiques sont en effet libérés dans des $V \varepsilon$ [15].

\section{Myélinisation axonale}

Les oligodendrocytes sont des cellules gliales en charge de la myélinisation des axones. La myéline, constituée 
de différentes protéines, forme une gaine autour des axones qui permet une transmission de l'information électrique beaucoup plus rapide au niveau des neurones. En l'absence de neurones, les VE oligodendrocytaires inhibent la formation de la gaine de myéline [16]. Mais quand les neurones sont présents, la libération de neurotransmetteurs excitateurs, suite à une stimulation électrique, peut entraîner, dans les oligodendrocytes périaxonaux, la fusion des corps multivésiculaires avec la membrane, ce qui conduit au relargage d'exosomes dans le milieu extracellulaire [17]. Des VE oligodendrocytaires contiennent des protéines constitutives de la myéline et des protéines associées à la protection contre le stress cellulaire [18]. Ces VE participeraient ainsi à la formation et au maintien de la gaine de myéline.

\section{Vésicules extracellulaires et maladies du système nerveux}

Ces dernières années, les $V \varepsilon$ sont apparues comme des actrices majeures dans la propagation des maladies neurodégénératives, dans la neuroinflammation du SN, et dans la tumorigenèse. Néanmoins, nous verrons qu'elles peuvent également avoir un rôle bénéfique pour faire face à ces maladies. Nous fournissons ici un bref aperçu de certaines des nouvelles données reliant les $V \varepsilon$ aux maladies neurologiques, comme la maladie d'Alzheimer, la sclérose en plaques ou le gliome (Figure 1, panneau inférieur).

\section{Les maladies neurodégénératives}

Les maladies neurodégénératives sont caractérisées par une mort progressive des cellules du SN. Les régions cérébrales et les types neuronaux touchés dépendent de la maladie neurodégénérative et expliquent la variété des symptômes. Depuis de nombreuses années, la formation d'agrégats intracellulaires de différentes protéines a été décrite.

Initialement décrite dans les maladies à prions [19], dont fait partie la maladie de Creutzfeldt-Jakob, la protéine prion intracellulaire anormalement repliée est capable de convertir la protéine prion normale en une protéine de forme anormale, induisant ainsi le processus d'agrégation protéique que l'on observe dans ces maladies. L'aggravation symptomatologique de la maladie est liée à la propagation de ces protéines anormales qui, une fois sécrétées dans le milieu extracellulaire, sont capturées par une autre cellule où la conversion et l'agrégation de la protéine prion se répète. Cette observation a conduit à penser que les $V \varepsilon$ pouvaient être impliquées dans la propagation de la maladie. $\varepsilon$ n effet, la protéine prion anormale est retrouvée associée aux VE [20] et la stimulation de la sécrétion vésiculaire augmente son transfert intercellulaire in vitro [21]. In vivo, l'injection de ces VE dans le cerveau de souris induit une maladie de type prion [22].

Ces premières données issues des maladies à prions ont conduit à l'hypothèse que le comportement de la protéine prion pourrait s'étendre à d'autres protéines impliquées dans certaines maladies neurodégénératives. La propagation de protéines anormalement repliées via les VE a ainsi été décrite dans la maladie de Parkinson [23], la sclérose latérale amyotrophique [24] et la maladie d'Alzheimer [25]. Dans cette dernière, deux lésions, liées à l'agrégation de deux protéines distinctes, coexistent : les peptides $\beta$-amyloïde $(A \beta)$, qui forment des plaques séniles extracellulaires, et la protéine tau, à l'origine des dégénérescences neurofibrillaires intracellulaires. Dans plusieurs modèles in vitro, il a été montré que la protéine tau pouvait être sécrétée dans des $V \varepsilon$ [26]. Des espèces particulières de protéines tau capables d'entraîner le mauvais repliement et l'agrégation de protéines tau normales, ont également été décrites dans des VE isolées à partir de cerveaux de souris transgéniques exprimant une protéine tau humaine mutée [27] et, plus récemment, à partir de cerveaux de patients [28]. Injectées dans le cerveau de souris, ces vésicules sont capables d'induire une maladie et participeraient à la propagation de celle-ci. La protéine tau est également sécrétée dans le milieu extracellulaire sous une forme non vésiculaire [28]. Cependant, les protéines tau présentes dans les $\checkmark \varepsilon$ semblent être plus efficaces pour déclencher les processus de conversion et d'agrégation [28]. Les VE microgliales participeraient également à la propagation des lésions dues à la protéine tau; l'inhibition de leur biogenèse ralentit en effet la progression de ces lésions in vivo [29].

Concernant les plaques séniles, des vésicules extracellulaires $(V E)$ dérivées de cerveaux de patients et contenant des oligomères de peptides $A \beta$ sont internalisées dans des cultures neuronales dérivées d'hIPSc et induisent une toxicité [30]. Les $V E$ astrocytaires accélèrent également l'agrégation des peptides $A \beta$ et empêchent leur clairance par la microglie in vitro. Le blocage de la sécrétion de ces vésicules entraîne une diminution de la formation des plaques et améliore la cognition in vivo [31].

Certaines études mettent cependant en évidence un rôle bénéfique des $V \varepsilon$ dans ces maladies. $\varepsilon n$ effet, des VE neuronales sont capables de se lier à des assemblages de peptides $A \beta$ et de les diriger vers la microglie afin de promouvoir leur dégradation et ainsi réduire les niveaux d'A $\beta$ extracellulaires [32]. Les peptides $A \beta$ sont capables d'inhiber la plasticité synaptique, mais des VE dérivées du liquide céphalo-spinal (LCS) humain permettent d'inverser cet effet. Dans ce cas, la présence, à la surface de ces $V \mathcal{E}$, de protéines se liant aux peptides $A \beta$ pourrait permettre à ces protéines de jouer un rôle de chélateur [33]. Ces données suggèrent que les VE pourraient contrecarrer l'action délétère des peptides $A \beta$ pathologiques, contribuant une nouvelle fois à la plasticité synaptique.

La compréhension du rôle des VE dans la physiopathologie des maladies neurodégénératives est donc primordiale. Celles-ci pourraient en effet être la cible de traitements permettant de ralentir la progression des 
maladies, voire de restaurer les fonctions physiologiques normales. La présence de VE dans les liquides biologiques, tels que le LCS et le plasma, conduit également à se questionner sur leur utilité comme biomarqueurs diagnostiques et pronostiques [34].

\section{Neuroinflammation dans un contexte de démyélinisation}

La sclérose en plaques (SEP) est une maladie inflammatoire du $S N$. Elle se caractérise par une démyélinisation des axones liée à un processus inflammatoire auquel participent des lymphocytes $B$ anormalement présents dans le tissu cérébral. Contrairement à ce qui est observé chez un sujet sain, des VE produites par des lymphocytes B isolés de patients atteints de SEP sont capables d'induire la mort d'oligodendrocytes, affectant ainsi la formation de la gaine de myéline [35]. Physiologiquement, ces lymphocytes sont uniquement présents dans les vaisseaux sanguins, et, de par l'existence de la barrière hémato-encéphalique $(B H \varepsilon)$, ils ne peuvent pas être en contact direct avec les cellules du SN. Toutefois, une altération de la perméabilité de la BHE accompagne souvent la neuroinflammation. Les VE sécrétées par les cellules endothéliales de la BHE pourraient jouer un rôle dans le processus d'extravasation des lymphocytes $B$ des vaisseaux sanguins vers le cerveau. Les VE produites par les lymphocytes $B$ contiennent, notamment, la protéine claudine-1. C'est elle qui va assurer la jonction entre les lymphocytes $B$ circulants et les cellules endothéliales de la BHE [36]. Une fois les lymphocytes adhérés à l'endothélium, leur migration dans le tissu cérébral est rendue possible par l'action de métalloprotéases et de la caspase-1, également sécrétées par les cellules endothéliales dans des $V \varepsilon$. Ces enzymes induisent une dégradation de la matrice extracellulaire rendant la BHE perméable [37].

Les cellules microgliales sont définies comme étant les «macrophages du cerveau » engagés dans la réponse inflammatoire pour défendre ce dernier des infections et réparer les tissus. Elles libèrent des $V \varepsilon$ qui ressemblent à celles produites par les lymphocytes B et les cellules dendritiques en conditions basales. En effet, elles contiennent des facteurs de signalisation liés à la réponse immunitaire, comme des molécules du complexe majeur d'histocompatibilité de classe II (CMH II), des protéines chaperones, des tétraspanines et des récepteurs membranaires. En contact avec des quantités élevées d'ATP extracellulaire, libéré par des astrocytes ou des tissus lésés, les cellules microgliales sécrètent des VE contenant de l'IL(interleukine)-1 $\beta$, une cytokine pro-inflammatoire, qui va agir directement au niveau de la lésion et contribuer à la signalisation immunitaire [38]. Dans un modèle murin de SEP, il a été montré que la microglie est capable de provoquer une réponse inflammatoire aberrante [39] résultant, en partie, de l'internalisation des $V \varepsilon$, et de leur cargo, par des neurones [40]. Ces travaux sont néanmoins controversés car une autre étude montre que l'injection de VE microgliales dans un modèle murin de SEP entraîne au contraire une amélioration de la motricité et une réduction des infiltrats inflammatoires, de la démyélinisation, et de la perte axonale [41]. Favorisant la migration et la maturation des cellules précurseurs d'oligodendrocytes, les VE microgliales participent cependant à la neuroprotection [42].

\section{Infiltration et croissance tumorale}

Les gliomes sont les cancers primitifs cérébraux les plus fréquents. Il en existe de nombreux types et sous-types. Leur pronostic dépend principalement du caractère limité ou infiltrant vis-à-vis du tissu cérébral. Dans la majorité des gliomes infiltrants, une infiltration tumorale dans les deux hémisphères cérébraux est observée au moment du diagnostic. Cela limite donc le traitement par résection chirurgicale complète puisque celui-ci n'est possible que pour les gliomes circonscrits. La capacité de migration des cellules tumorales est étroitement liée à l'interaction que ces cellules ont avec le tissu qu'elles envahissent. En raison de leur capacité à moduler d'autres cellules, les $V \varepsilon$ ont été largement étudiées dans le contexte des tumeurs et de l'environnement tumoral. Dans les gliomes infiltrants, les interactions entre cellules tumorales et cellules du microenvironnement sont réciproques. Des $V \varepsilon$ issues de cellules gliales tumorales sont capables d'activer des astrocytes non tumoraux, provoquant des modifications de la matrice extracellulaire, facilitant ainsi l'invasion du tissu par le gliome [43-45]. La croissance tumorale est également très dépendante de la néo-angiogenèse. $\varepsilon n$ conditions hypoxiques, les $V \varepsilon$ d'origine tumorale stimulent la néo-vascularisation en agissant sur les cellules endothéliales [46]. Une analyse par spectrométrie de masse des $V \varepsilon$ sécrétées par des cellules gliales tumorales a identifié plus de 1000 protéines ayant des caractéristiques angiogéniques et invasives [47]. Le glioblastome, la tumeur la plus agressive du SN, libère des VE qui contiennent des micro-ARN, des ARNm, des histones et diverses protéines qui peuvent affecter la croissance tumorale [48]. Le transfert vésiculaire d'un micro-ARN spécifique, le miR-1238, d'une cellule cancéreuse à une autre, a également été impliqué dans la chimiorésistance [49]. À l'inverse, il a été montré que des $V \varepsilon$ microgliales, testées sur une culture de cellules isolées de gliome, étaient capables d'inhiber l'invasion tumorale au cours du temps, soulignant cette fois-ci un rôle bénéfique des VE [50].

\section{Conclusions}

Nous avons dressé un aperçu de l'importance et de la diversité des échanges intercellulaires vésiculaires pour le fonctionnement normal du système nerveux, bien que ce soit dans des conditions pathologiques que les vésicules extracellulaires (VE) aient été les plus étudiées. En effet, de par leur participation aux processus pathologiques, celles-ci pourraient être utilisées pour le diagnostic de maladies cérébrales ou être la cible de thérapies innovantes. Toutefois, elles peuvent avoir un 
rôle aussi bien néfaste que bénéfique. Il est donc primordial d'identifier les sous-types vésiculaires impliqués dans la progression des maladies et ceux impliqués dans le maintien des fonctions physiologiques, afin de développer des outils thérapeutiques ciblant uniquement les VE néfastes. Une autre approche consisterait à les utiliser non pas comme cible mais comme agent thérapeutique afin d'accroître leur rôle bénéfique.

Notons cependant que les données actuellement disponibles ont été principalement obtenues à partir de cultures cellulaires in vitro qui ne sont pas forcément représentatives des mécanismes réels qui existent dans un système biologique complexe comme le cerveau. En effet, l'impact fonctionnel des $V \varepsilon$ au sein des réseaux cellulaires du cerveau reste difficile à établir compte tenu de la complexité des populations cellulaires impliquées dans la libération et l'internalisation des $V \varepsilon$, ainsi que des limites techniques des approches actuelles de leur analyse. Ces données ouvrent cependant la voie à un champ d'étude très vaste pour mieux comprendre le fonctionnement cérébral global ainsi que pour développer de nouveaux outils thérapeutiques. $\diamond$

\section{SUMMARY}

\section{Extracellular vesicles in the central nervous system}

Extracellular Vesicles ( $\varepsilon V s$ ) are released by a wide diversity of cells. They contain proteins, RNAs and lipids that will be exchanged between these cells. They represent therefore a major form of intercellular communication in both physiological and pathological conditions. This is particularly relevant in the nervous system where neurons and glial cells form a very dense network where billions of connections are made. In this review, the different roles played by the $E V s$ in a healthy brain to maintain cerebral homeostasis during development, synaptic transmission or axonal myelination will be discussed. In addition, the pathological aspects of $E V$ s presence will also be addressed. In recent years, the EVs have emerged as major players in the spread of neurodegenerative diseases, in neuroinflammation and in tumor development, although they may also be beneficial in some conditions. $\diamond$

\section{LIENS D'INTÉRÊT}

Les auteurs déclarent n'avoir aucun lien d'intérêt concernant les données publiées dans cet article.

\section{RÉFÉRENCES}

1. Sharma P, Mesci P, Carromeu C, et al. Exosomes regulate neurogenesis and circuit assembly. Proc Natl Acad Sci USA 2019 ; 116 : 16086-94.

2. Wang $S$, Cesca F, Loers G, et al. Synapsin I Is an Oligomannose-Carrying Glycoprotein, Acts As an Oligomannose-Binding Lectin, and Promotes Neurite Outgrowth and Neuronal Survival When Released via Glia-Derived Exosomes. J Neurosci 2011 ; 31 : 7275-90.

3. Chaudhuri AD, Dasgheyb RM, DeVine LR, et al. Stimulus-dependent modifications in astrocytederived extracellular VEicle cargo regulate neuronal excitability. Glia $2020 ; 68$ : 128-44.

4. Lemaire $Q$, Raffo-Romero A, Arab T, et al. Isolation of microglia-derived extracellular Vesicles: towards miRNA signatures and neuroprotection. J Nanobiotechnol $2019 ; 17: 119$.

5. Chivet M, Javalet C, Laulagnier $K$, et al. Exosomes secreted by cortical neurons upon glutamatergic synapse activation specifically interact with neurons. J Extracell Vesicles $2014 ; 3: 24722$.

6. Fauré J, Lachenal G, Court M, et al. Exosomes are released by cultured cortical neurones. Mol Cell Neurosci $2006 ; 31: 642-8$.

7. Lachenal G, Pernet-Gallay K, Chivet M, et al. Release of exosomes from differentiated neurons and its regulation by synaptic glutamatergic activity. Mol Cell Neurosci2011; 46: 409-18.

8. Antonucci F, Turola $\varepsilon$, Riganti L, et al. MicroVesicles released from microglia stimulate synaptic activity via enhanced sphingolipid metabolism: Microglial MVs increase sphingolipid metabolism in neurons. EMBO J $2012 ; 31: 1231-40$.
9. Gabrielli M, Battista N, Riganti L, et al. Active endocannabinoids are secreted on extracellular membrane Vesicles. EMBO Rep 2015 ; 16 : 213-20.

10. Holm MM, Kaiser J, Schwab ME. Extracellular Vesicles: Multimodal Envoys in Neural Maintenance and Repair. Trends Neurosci 2018 ; 41 : 360-72.

11. Korkut C, Li Y, Koles K, et al. Regulation of Postsynaptic Retrograde Signaling by Presynaptic Exosome Release. Neuron $2013 ; 77$ : 1039-46.

12. Bahrini I, Song J, Diez D, et al. Neuronal exosomes facilitate synaptic pruning by up-regulating complement factors in microglia. Sci Rep $2015 ; 5: 7989$.

13. Chivet $M$, Javalet $C$, Hemming $F$, et al. Exosomes as a novel way of interneuronal communication. Biochem Soc Trans $2013 ; 41: 241-4$.

14. Valadi $H$, Ekström $K$, Bossios $A$, et al. Exosome-mediated transfer of mRNAs and microRNAs is a novel mechanism of genetic exchange between cells. Nat Cell Biol $2007 ; 9$ : 654-9.

15. Goldie BJ, Dun MD, Lin M, et al. Activity-associated miRNA are packaged in Maplb-enriched exosomes released from depolarized neurons. Nucleic Acids Res $2014 ; 42$ : 9195-208.

16. Bakhti M, Winter C, Simons M. Inhibition of Myelin Membrane Sheath Formation by Oligodendrocyte-derived Exosome-like Vesicles. J Biol Chemstry $2011 ; 286: 787-96$.

17. Frühbeis C, Fröhlich D, Kuo WP, et al. Neurotransmitter-Triggered Transfer of Exosomes Mediates Oligodendrocyte-Neuron Communication. PLoS Biol $2013 ; 11$ : e1001604.

18. Krämer-Albers $\varepsilon-M$, Bretz N, Tenzer $S$, et al. Oligodendrocytes secrete exosomes containing major myelin and stress-protective proteins: Trophic support for axons? Prot Clin Appl 2007 ; 1 : 1446-61.

19. Prusiner SB. Novel proteinaceous infectious particles cause scrapie. Science $1982 ; 216: 136-44$.

20. Fevrier $B$, Vilette $D$, Archer $F$, et al. Cells release prions in association with exosomes. Proc Natl Acad Sci U S A 2004 ; 101 : 9683-8.

21. Guo BB, Bellingham SA, Hill AF. Stimulating the Release of Exosomes Increases the Intercellular Transfer of Prions. J Biol Chem 2016 ; 291 : 5128 37.

22. Vella LJ, Sharples RA, Lawson VA, et al. Packaging of prions into exosomes is associated with a novel pathway of PrP processing. J Pathol 2007 ; 211 : 582-90.

23. Ma J, Gao J, Wang J, et al. Prion-Like Mechanisms in Parkinson's Disease. Fron. Neurosci $2019 ; 13: 552$.

24. McAlary L, Plotkin SS, Yerbury JJ, et al. Prion-Like Propagation of Protein Misfolding and Aggregation in Amyotrophic Lateral Sclerosis. Front Mol Neurosci $2019 ; 12: 262$.

25. Colin M, Dujardin S, Schraen-Maschke S, et al. From the prionlike propagation hypothesis to therapeutic strategies of anti-tau immunotherapy. Acta Neuropathol $2020 ; 139: 3-25$.

26. Pérez M, Avila J, Hernández F. Propagation of Tau via Extracellular Vesicles. Front Neurosci $2019 ; 13: 698$.

27. Polanco JC, Scicluna BJ, Hill AF, et al. Extracellular vesicles Isolated from the Brains of rTg4510 Mice Seed Tau Protein Aggregation in a Thresholddependent Manner. J Biol Chem 2016 ; 291 : 12445-66.

28. Ruan Z, Pathak D, Venkatesan Kalavai S, et al. Alzheimer's disease brainderived extracellular vesicles spread tau pathology in interneurons. Brain $2021 ; 144: 288-309$

29. Asai H, Ikezu S, Tsunoda S, et al. Depletion of microglia and inhibition of exosome synthesis halt tau propagation. Nat Neurosci $2015 ; 18: 1584-93$.

30. Sardar Sinha M, Ansell-Schultz A, Civitelli L, et al. Alzheimer's disease pathology propagation by exosomes containing toxic amyloid-beta oligomers. Acta Neuropathol 2018 ; $136: 41-56$.

31. Dinkins MB, Enasko J, Hernandez C, et al. Neutral Sphingomyelinase-2 Deficiency Ameliorates Alzheimer's Disease Pathology and ImproVE Cognition in the 5XFAD Mouse. J Neurosci 2016 ; 36 : 8653-67.

32. Yuyama K, Sun H, Mitsutake S, et al. Sphingolipid-modulated Exosome Secretion Promotes Clearance of Amyloid- $\beta$ by Microglia. J Biol Chem 2012 ; 287 : 10977-89.

33. An K, Klyubin I, Kim Y, et al. Exosomes neutralize synaptic-plasticitydisrupting activity of $A \beta$ assemblies in vivo. Mol Brain $2013 ; 6: 47$.

34. Gámez-Valero $A$, Beyer $K$, Borràs $F \varepsilon$. Extracellular Vesicles, new actors in the search for biomarkers of dementias. Neurobiol Aging 2019; $74: 15-20$.

35. Benjamins JA, Nedelkoska L, Touil H, et al. Exosome-enriched fractions from MS B cells induce oligodendrocyte death. Neurol Neuroimmunol Neuroinflamm $2019 ; 6$ : e550.

36. Paul D, Baena V, Ge S, et al. Appearance of claudin-5+ leukocytes in the central nervous system during neuroinflammation: a novel role for endothelial-derived extracellular Vesicles. J Neuroinflammation 2016; 13 : 292. 


\section{RéFÉRENCES}

37. Sáenz-Cuesta M, Osorio-Querejeta I, Otaegui D. Extracellular Vesicles in Multiple Sclerosis: What are They Telling Us? Front Cell Neurosci $2014 ; 8: 100$.

38. Bianco F, Pravettoni $\varepsilon$, Colombo A, et al. Astrocyte-Derived ATP Induces VEicle Shedding and IL-1 $\beta$ Release from Microglia. J Immunol 2005 ; 174 : 7268-77.

39. Zrzavy T, Hametner S, Wimmer I, et al. Loss of 'homeostatic' microglia and patterns of their activation in active multiple sclerosis. Brain $2017 ; 140: 1900-13$.

40. Prada I, Gabrielli M, Turola $\varepsilon$, et al. Glia-to-neuron transfer of miRNAs via extracellular Vesicles: $a$ new mechanism underlying inflammation-induced synaptic alterations. Acta Neuropathol 2018 ; $135: 529-50$.

41. Casella G, Colombo F, Finardi A, et al. Extracellular Vesicles Containing IL-4 Modulate Neuroinflammation in a Mouse Model of Multiple Sclerosis. Mol Ther 2018 ; 26 : 2107-18.

42. Williams JL, Gatson NN, Smith KM, et al. Serum exosomes in pregnancy-associated immune modulation and neuroprotection during CNS autoimmunity. Clin Immunoly 2013 ; 149 : 236-43.

43. D’Asti $\varepsilon$, Garnier D, Lee TH, et al. Oncogenic extracellular Vesicles in brain tumor progression. Front Physiol $2012 ; 3: 294$.

44. Hallal S, Mallawaaratchy DM, Wei H, et al. Extracellular Vesicles Released by Glioblastoma Cells Stimulate Normal Astrocytes to Acquire a Tumor-Supportive Phenotype Via p53 and MyC Signaling Pathways. Mol Neurobiol 2019 ; 56 : 4566-81.
45. Oushy S, Hellwinkel JE, Wang M, et al. Glioblastoma multiforme-derived extracellular Vesicles drive normal astrocytes towards a tumour-enhancing phenotype. Philos Trans R Soc Lond B Biol Sci 2018 ; 373 : 20160477.

46. Tadokoro H, Umezu T, Ohyashiki K, et al. Exosomes Derived from Hypoxic Leukemia Cells Enhance Tube Formation in Endothelial Cells. J Biol Chem $2013 ; 288: 34343-51$

47. Jaiswal R, Sedger LM. Intercellular VEicular Transfer by Exosomes, Microparticles and Oncosomes- Implications for Cancer Biology and Treatments. Front Oncol $2019 ; 9$ : 125.

48. Skog J, Wurdinger T, Rijn S van, et al. Glioblastoma microVesicles transport RNA and protein that promote tumor growth and provide diagnostic biomarkers. Nat Cell Biol $2008 ; 10: 1470-6$.

49. Yin J, Zeng $A$, Zhang Z, et al. Exosomal transfer of miR-1238 contributes to temozolomide-resistance in glioblastoma. EBioMedicine $2019 ; 42$ : 238-51.

50. Murgoci A-N, Cizkova D, Majerova P, et al. Brain-Cortex Microglia-Derived Exosomes: Nanoparticles for Glioma Therapy. ChemPhysChem 2018 ; 19 : 1205-14

\section{TIRÉS À PART}

M. Colin

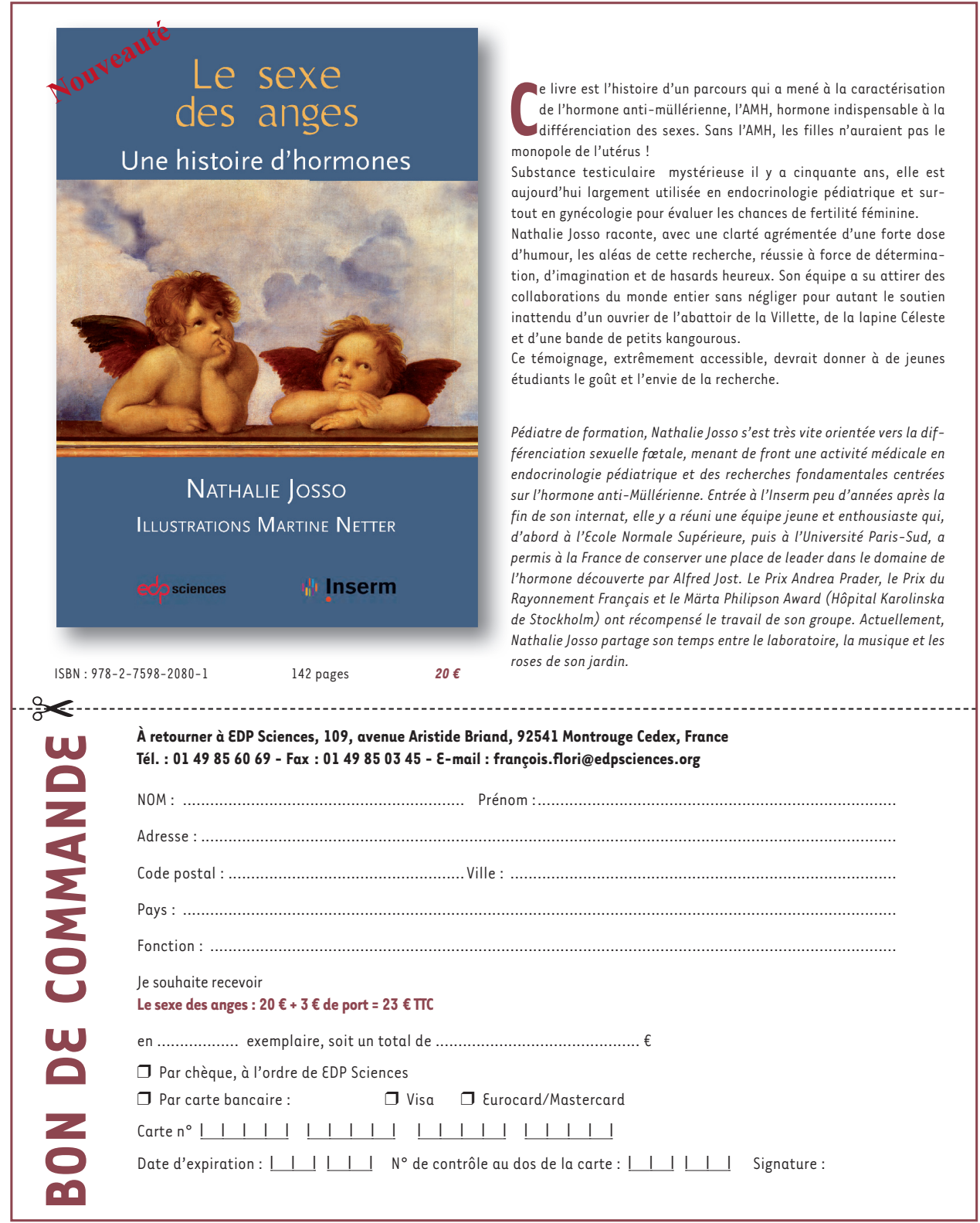

